\title{
Determination of viscoelastic coefficients from the optical transmission of a planar liquid crystal cell with low-frequency modulated voltage
}

\author{
H. Schmiedel, R. Stannarius, and M. Grigutsch \\ Fachbereich Physik der Universität Leipzig, 04103 Leipzig, Germany \\ R. Hirning, J. Stelzer, and H.-R. Trebin \\ Institut für Theoretische und Angewandte Physik, Universität Stuttgart, Institut fur Theoretische \\ und Angewandte Physik, 70550 Stuttgart, Germany
}

(Received 17 May 1993; accepted for publication 19 July 1993)

\begin{abstract}
The viscoelastic response of the nematic director field to low-frequency modulations of the driving voltage was studied by means of optical transmission measurements. An external ac voltage above the critical field $U_{c}$ was weakly amplitude modulated with frequencies in the range of 1 to $100 \mathrm{~Hz}$. The viscosity coefficients $\alpha_{1}, \gamma_{1}, \gamma_{2}$, and $\eta_{b}$ influence the time dependence of the director field. They were determined by fitting the phase and amplitude of theoretically calculated optical transmission curves to measured data.
\end{abstract}

\section{INTRODUCTION}

The dynamical behavior of liquid crystals is described by the Leslie-Ericksen equations. In three-dimensional geometry, there are six Leslie viscosity coefficients, five of them being independent. In a one-dimensional geometry, the motion depends on four viscosities only. The examination of the electro-optical switching of thin planar cells near the Fréedericksz threshold $U_{c}$ provides the rotational viscosity $\gamma_{1}$. The other viscosities cannot be obtained in such an easy way. There are, however, alternative experimental methods that allow a more or less direct determination, too. ${ }^{1-6}$

Our intention has been to find a method to measure all four viscosity coefficients in a simple experimental setup. We modified an existing device for measuring elastic constants ${ }^{7}$ by changing the driving ac voltage from a constant effective value to a weakly modulated voltage, and we performed time dependent measurements.

If the voltage $U$ of a planar nematic cell is changed sufficiently slowly, the director field in the cell follows the changes of $U$ adiabatically in a state of stationary equilibrium (free energy minimum). We denote this case as "quasistatic." Thus, one can determine the elastic constants $K_{11}$ and $K_{33}$. If the amplitude of a voltage $U>U_{c}$ is modulated with a period comparable to the characteristic switching time of the cell, the director response is delayed with respect to the changes of the electric field and the amplitude of the director reorientation decreases. This fact is reflected by a phase shift between the optical transmission and the electrical input, and by a decrease of the modulation amplitude of the transmission function. Normally, nematic LC displays are driven by electric fields of frequencies within the order of $\mathrm{kHz}$. At such frequencies, the director field in the display cell experiences the effective value of the applied voltage only, because the nematic is too viscous to follow the rapidly alternating (e.g., sinusoidal) electric field.

The frequency dependence of amplitude and phase of the optical transmission curve provides information on all four viscosity coefficients mentioned above. If $U$ is switched on or off between zero and a voltage slightly above $U_{c}$, an independent method for the determination of $\gamma_{1}$ is applicable to the same system.

\section{EXPERIMENTAL SETUP}

The typical electro-optical characteristic of a planar cell is shown in Fig. 1. The diagram was recorded quasistatically. With increasing effective voltage, the director field is deformed gradually from planar to almost homeotropic orientation. The optical transmission of the cell with crossed polarizers in $45^{\circ}$ orientation to the surface alignment is determined by the interference of the ordinary wave subject to the index of refraction $n_{o}$ and the extraordinary wave which experiences an effective index $n_{\text {eff }}$. The latter decreases with increasing director deformation from $n_{e}$ to $n_{o}$, and the optical transmission passes through several interference maxima and minima.

The cells were illuminated with monochromatic light, $\lambda=632.8 \mathrm{~nm}$, by a laser source at normal incidence. The integral transmission intensity over an active spot of approximately $1 \mathrm{~mm}^{2}$ was measured by a photomultiplier and was recorded digitally with a homebuilt setup. Finally, data were transmitted to a PC for processing and storage.

The sample cells were made from ITO-coated glass plates with an orienting layer of $60^{\circ}$ obliquely evaporated SiO. This surface preparation provides planar alignment of the director at the glass with strong anchoring. The cells were filled with the nematic mixture ZLI 2293 (Merck), whose clearing point is between 81 and $85^{\circ} \mathrm{C}$. Cells of thicknesses between 10 and $40 \mu \mathrm{m}$ have been prepared with different spacer foils. The cell thickness has been determined from interference patterns by infrared transmission spectroscopy of the empty cells.

For the study of the dynamics of the liquid crystal, we chose an offset voltage $U_{0}$ so that the static transmission was approximately in the middle of one of the slopes of the interference curve. We modulated the voltage at the electrodes with a low frequency $f_{m}(1 \ldots 100 \mathrm{~Hz})$ and an amplitude $U_{m}$ (Fig. 1): 


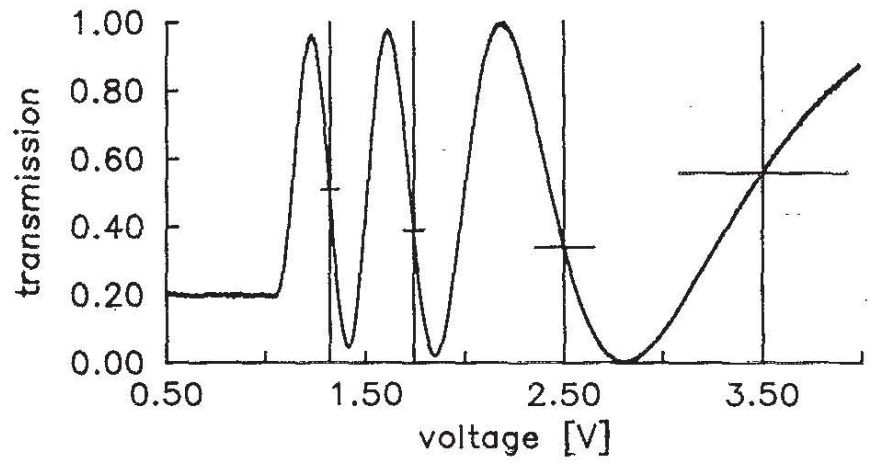

FIG. 1. Experimental static transmission of a planar cell as function of applied effective voltage, crossed polarizers in $45^{\circ}$ position. Vertical bars: offset voltages $U_{0}$ chosen in the modulation experiments. Horizontal bars: modulation amplitudes $U_{m}$. Nematic substance: ZLI 2293 (Merck), $T_{\text {red }}=0.906\left(51.5^{\circ} \mathrm{C}\right)$.

$$
U_{\text {eff }}(t)=U_{0}+U_{m} \cos \left(2 \pi f_{m} t\right) .
$$

The modulation amplitude was small enough to provoke a linear response of the optical transmission to changes in $U$. However, our calculations provide an exact solution of the Leslie-Ericksen equations, and the optical characteristics are computed by the $4 \times 4$ matrix formalism. Therefore, there are no limiting assumptions in the theoretical fitting procedure, and linearity of the transmission response function is not required. In principle, any modulation offset and amplitude could be applied, but choosing a point of linear slope of the static curve and small modulation amplitudes makes the fitting procedure much easier.

In practice, we used a constant carrier frequency of 1 $\mathrm{kHz}$ in all experiments. Only its effective value was modulated. This frequency is fast enough to avoid dc effects in the sample. The director is sensitive to the effective value of the carrier. Furthermore, the dielectric anisotropy $\Delta \epsilon$ is slightly frequency dependent. With a constant carrier frequency we can use the value of $\Delta \epsilon$ for $1 \mathrm{kHz}$ in all experiments.

Due to the viscosity of liquid crystals, the optical transmission for a modulated external electric field follows the static characteristics of Fig. 1 only at very low frequencies. With increasing $f_{m}$, it deviates from the quasistatic curve, but after a short time the transmission versus voltage becomes a stationary Lissajous figure (Fig. 2). This

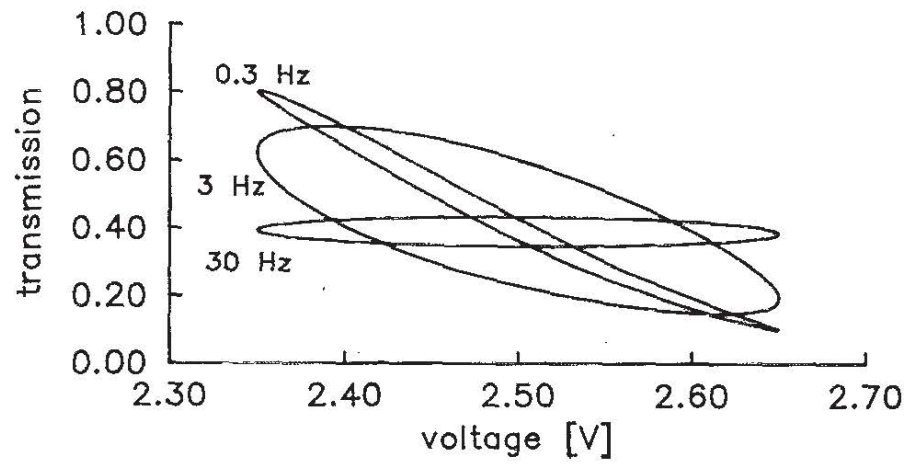

FIG. 2. Trānsmission vs voltage for different modulation frequencíes. Nematic substance: ZLI 2293 (Merck), $T_{\text {red }}=0.906\left(51.5^{\circ} \mathrm{C}\right.$ ). curve was recorded with a device normally used for measuring elastic constants. ${ }^{7}$ In each experiment, we have recorded 16 cycles of the stationary figures and averaged them to one cycle. Subsequent runs with the same offset and modulation resulted in reproducible and stable Lissajous figures.

\section{THEORY}

Take a nematic layer of thickness $d$ with the layer normal coinciding with the $z$ axis of a Cartesian coordinate system. At both surfaces of the layer, the director is anchored parallel to the $x$ axis. Thus the azimuthal angle is constant, and the director can be described by a tilt angle $\theta(z, t)$ only. $\theta$ is measured with respect to the $x$ axis. The motion of the director is coupled to a macroscopic mass flux $v$ in the $x$ direction (backflow ${ }^{8}$ ).

The evolution of $\theta$ and $v$ under an applied voltage $U_{\text {eff }}(t)$ is described by a system of coupled partial differential equations, the Leslie-Ericksen equations: ${ }^{9,10}$

$$
\begin{aligned}
& \sigma^{\langle z x\rangle}=f_{1}(\theta) v_{z}-f_{2}(\theta) \dot{\theta}, \\
& \gamma_{1} \dot{\theta}=f_{3}(\theta) \theta_{z z}+\frac{1}{2} f_{3}^{\prime}(\theta) \theta_{z}^{2}+f_{4}(\theta) E^{2}+f_{2}(\theta) v_{z},
\end{aligned}
$$

with

$$
\begin{aligned}
& f_{1}(\theta)=\frac{1}{4} \alpha_{1} \sin ^{2} 2 \theta+\eta_{b}-\gamma_{2} \sin ^{2} \theta, \\
& f_{2}(\theta)=\gamma_{2} \sin ^{2} \theta-\frac{1}{2}\left(\gamma_{1}+\gamma_{2}\right), \\
& f_{3}(\theta)=K_{11} \cos ^{2} \theta+K_{33} \sin ^{2} \theta, f_{3}^{\prime}(\theta)=\frac{d f_{3}(\theta)}{d \theta}, \\
& f_{4}(\theta)=\frac{1}{2} \epsilon_{0}\left(\epsilon_{\|}-\epsilon_{1}\right) \sin 2 \theta, \\
& \dot{\theta}=\frac{\partial \theta}{\partial t}, \quad \theta_{z}=\frac{\partial \theta}{\partial z}, \quad \theta_{z z}=\frac{\partial^{2} \theta}{\partial z^{2}}, \quad v_{z}=\frac{\partial v}{\partial z} .
\end{aligned}
$$

$\sigma^{\langle z x\rangle}$ is the $z x$ component of the nematic strain tensor; $E$ is the $z$ component of the electric field:

$$
\begin{aligned}
E(z, t)= & \frac{U_{\text {eff }}(t)}{\left(\epsilon_{\|} \cos ^{2} \theta+\epsilon_{\perp} \sin ^{2} \theta\right)} \\
& \times\left(\int_{0}^{d} \frac{d z}{\epsilon_{\|} \cos ^{2} \theta+\epsilon_{\perp} \sin ^{2} \theta}\right)^{-1} .
\end{aligned}
$$

Finally, $K_{11}$ and $K_{33}$ are the Frank elastic constants for splay and bend deformation, respectively. $\gamma_{1}$ and $\gamma_{2}$ are the rotational viscosities, and $\eta_{b}$ is the shear viscosity. The viscosities $\gamma_{1}, \gamma_{2}$ and $\eta_{b}$ can be expressed by the Leslie coefficients $\alpha_{1} \ldots \alpha_{6}$ :

$\gamma_{1}=\alpha_{3}-\alpha_{2}, \quad \gamma_{2}=\alpha_{3}+\alpha_{2}, \quad \eta_{b}=\frac{1}{2}\left(\alpha_{4}+\alpha_{3}+\alpha_{6}\right)$.

Following the work of van Doorn, ${ }^{11}$ in Eqs. (1) and (2) we neglected the inertial terms containing second order time derivatives of $\theta$.

The Eqs. (1) and (2) are subject to the obvious boundary conditions for strong anchoring:

$$
\begin{aligned}
& \theta(z=0, t)=\theta(z=d, t)=0 \text { and } \\
& v(z=0, t)=v(z=d, t)=0 .
\end{aligned}
$$




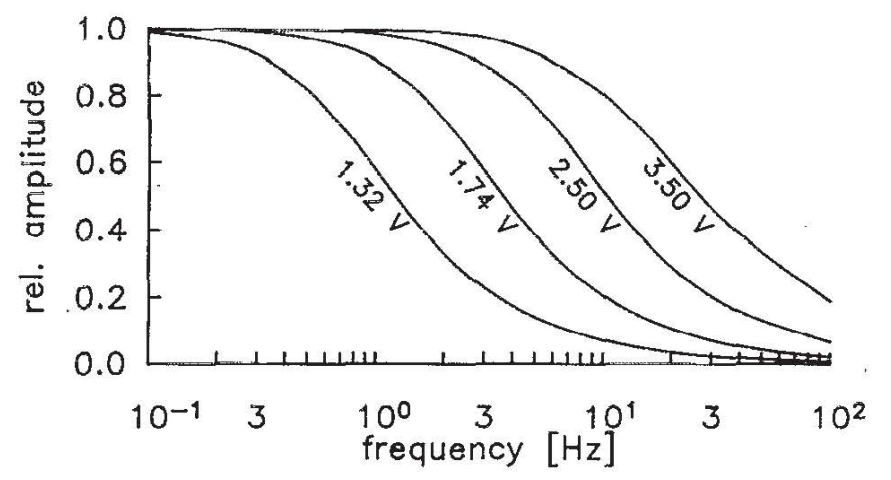

FIG. 3. Relative amplitudes of the response for the offset voltages given in Fig. 1. The amplitude for frequency 0 is used as reference.

By eliminating $v_{z}$ one arrives at the equation:

$$
\gamma_{\mathrm{eff}} \dot{\theta}=f_{3} \theta_{z z}+\frac{1}{2} f_{3}^{\prime} \theta_{z}^{2}+f_{4} E^{2}+\sigma^{\langle z x\rangle} \frac{f_{2}}{f_{1}}
$$

which is similar to Eq. (2) and thus can be interpreted in terms of an effective rotational viscosity $\gamma_{\text {eff }}$, where

$$
\gamma_{\mathrm{eff}}(\theta)=\gamma_{1}-\frac{f_{2}^{2}}{f_{1}}
$$

After solving the Leslie-Ericksen equations numerically, the theoretical transmission curve for monochromatic light passing through the layer between crossed polarizers is obtained as a function of viscosities and elastic constants.

Starting from an arbitrary director deformation, the solution of the above system of differential equations for a low-frequency modulated external electric field leads to a stationary cyclic behavior of the director field. The cyclic optical transmission function corresponding to the director field is determined by application of geometrical optics.

$$
I=I_{0} \sin ^{2}\left(\pi\left(n_{\mathrm{eff}}-n_{o}\right) \frac{d}{\lambda}\right)
$$

is the transmission function which depends on the director field via the effective index of refraction

$$
n_{\mathrm{eff}}=\int_{0}^{d} \frac{n_{\mathrm{e}} n_{o}}{\sqrt{\left[n_{o}^{2} \cos ^{2}(\theta)+n_{e}^{2} \sin ^{2}(\theta)\right]}} d z .
$$

In the case of normal incidence of light without any twist in the director field Eq. (6) above, coming from pure geometrical optics, and the $4 \times 4$-matrix method we used in the calculation yield the same results.

The response of the liquid crystal to a low-frequency modulated voltage (Figs. 3 and 4) can be used to determine visco-elastic material parameters by fitting the simulated transmission to the measured one for a set of experiments. Keeping the temperature constant, experiments can be performed at different slopes of the transmission curve (see Fig. 1). At offset voltages $U_{0}$ near the Freedericksz threshold $U_{c}$, a good approximation to $\gamma_{\text {eff }}$ is given by $\eta_{\text {splay }}$, where

$$
\eta_{\text {splay }}=\gamma_{\text {eff }}(\theta=0)=\gamma_{1}-\frac{\alpha_{3}^{2}}{\eta_{b}}
$$

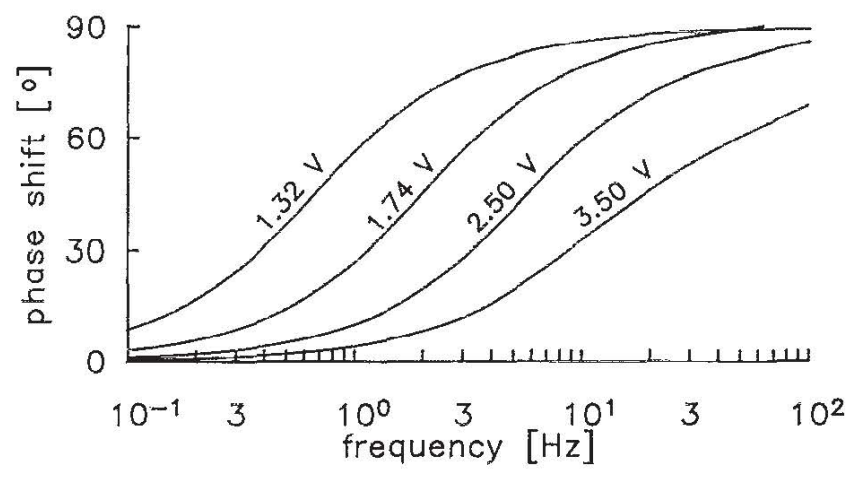

FIG. 4. Phase shift between the response and the driving voltage for the offset voltages given in Fig. 1.

Because for elongated molecules $\left|\alpha_{3}\right|<\left|\alpha_{2}\right|$ and hence $\gamma_{\text {eff }} \approx \gamma_{1}$, we expect good values for $\gamma_{1}$. At higher offset voltages $\left(U_{0}>U_{c}\right)$, the director is more aligned with the electric field than for low voltages. Thus

$$
\eta_{\text {bend }}=\gamma_{\text {eff }}\left(\theta=\frac{\pi}{2}\right)=\gamma_{1}-\frac{\alpha_{2}^{2}}{\eta_{b}-\gamma_{2}}
$$

is a good approximation to $\gamma_{\text {eff }}$ and we expect good values for $\gamma_{2}, \eta_{b}$ and $\alpha_{1}$, too.

At each slope of the transmission versus voltage curve, i.e., at each offset voltage, a set of experiments was performed with different modulation frequencies, starting with very slow modulations where the director follows the field changes immediately, up to high modulation frequencies where the amplitude modulation of the optical transmission has decayed to zero. For all these measurements at constant temperature, one set of parameters $\gamma_{1}, \gamma_{2}, \alpha_{1}$, and $\eta_{b}$ was found that fits the experimental curves. This is the advantage of the method over a simple ON or OFF switching of the cell, where in principle all these parameters are involved, too, but-even by varying the voltages of the switching processes-it is difficult to select information about the different viscosity parameters.

\section{RESULTS AND DISCUSSION}

From the static transmission curve of the cell used (Fig. 1), the elastic constants $K_{11}$ and $K_{33}$ were determined

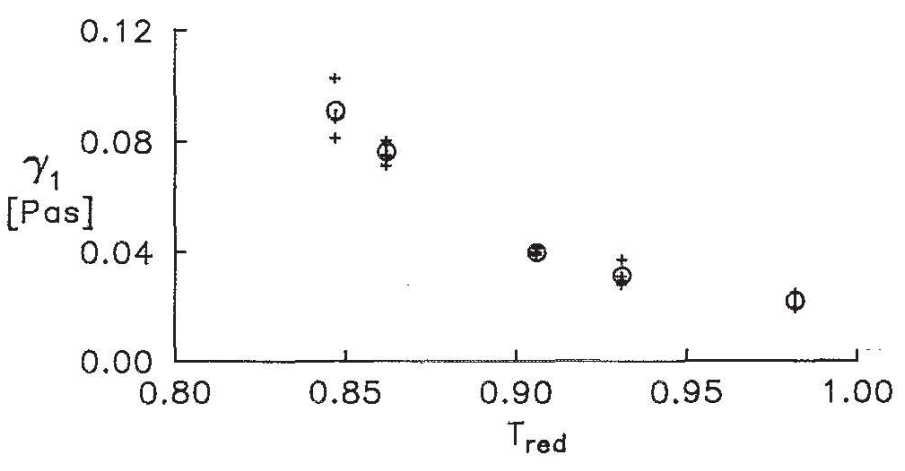

FIG. 5. Rotational viscosity $\gamma_{1}$. The crosses are values obtained by fitting the calculated transmission to the measured ones for several offset voltages. The circles give the average values. 


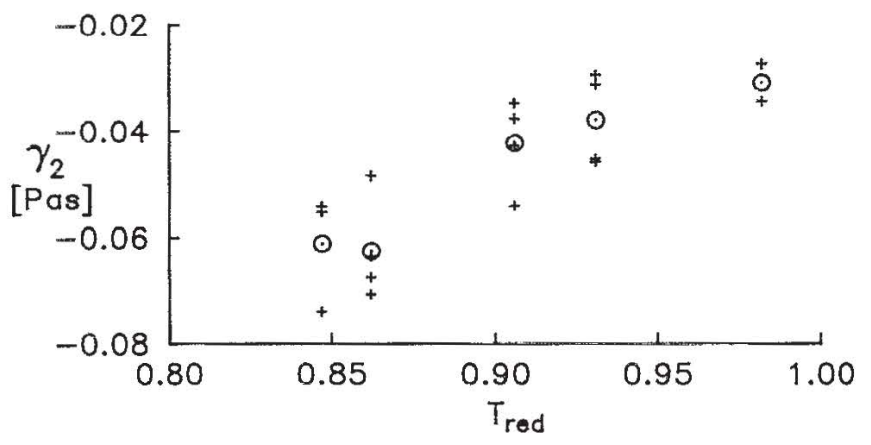

FIG. 6. Rotational viscosity $\gamma_{2}$. The crosses are values obtained by fitting the calculated transmission to the measured ones for several offset voltages. The circles give the average values.

with the method described in Ref. 7. The difference between the diffraction indices $\Delta n$ is related to the transmission of light with wavelength $\lambda$ at zero voltage, where the director pattern is homogeneous according to Eq. (6).

The initial intensity of the transmitted light $I_{0}$ corresponds to the maximum order of interference. As $\lambda, n_{e}$, and $n_{o}$ are known with sufficient precision, Eq. (6) provides a control of the cell thickness measurement.

From the fit of the frequency dependence of the amplitudes and phases of the optical transmission curves, we have determined the viscosity coefficients in dynamic experiments. At each temperature, experimental Lissajous figures of the $I(U)$ dependence have been recorded at a set of frequencies $f_{m}$ and for different offset voltages $U_{0}$. The modulation amplitudes $U_{m}$ were kept small enough to stay in the linear slopes of the transmission curve. The vertical bars in Fig. 1 show the corresponding values of $U_{0}$ and $U_{m}$ at one particular temperature. As we expected, the transition range from the low-frequency regime where the director follows the voltage function without delay and without loss in modulation amplitude to the high-frequency regime where the director averages the input modulation to an effective value shifts with increasing offset voltage. The transition frequency increases with higher offset voltage. This is reflected by Figs. 3 and 4 where the measured data are presented. The amplitudes decay from the value corresponding to the static curve down to zero, while the phase

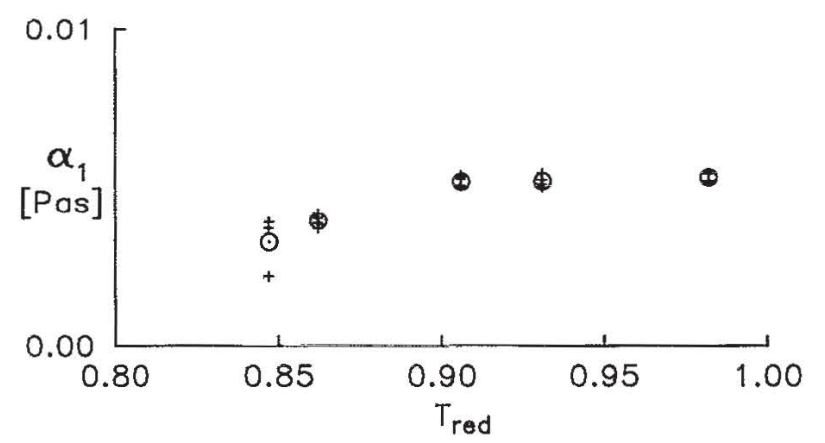

FIG. 7. Leslie viscosity coefficient $\alpha_{1}$. The crosses are values obtained by fitting the calculated transmission to the measured ones for several offset voltages. The circles give the average values.

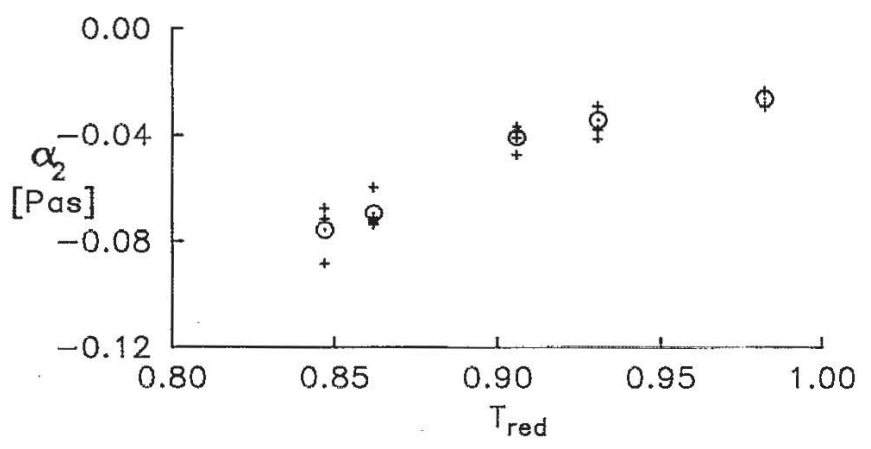

FIG. 8. Leslie viscosity coefficient $\alpha_{2}$ calculated using Eq. (3). The crosses are values obtained by fitting the calculated transmission to the measured ones for several offset voltages. The circles give the average values.

shift of the Lissajous figure increases from zero to $\pi / 2$. This is the behavior commonly known from a damped oscillator without inertial term. The resonance frequency is determined by the damping term from the viscous torques and the electrical and elastic torques that drive the director field. With increasing offset voltage, the latter torques increase approximately proportional to $U_{0}^{2}$, and the effective viscosities decrease due to flow coupling, which leads to a noticeable rise in the transition frequency.

Our method provides an easy tool for the determination of the dynamic characteristics of a nematic cell. It is not restricted to planar cells but could as well be applied to any other configuration like TN, STN, homeotropic, or hybrid cells.

The simulated curves of the optical transmission versus voltage curves were fitted to the experimental data with a multidimensional minimization algorithm varying the parameters $\gamma_{1}, \gamma_{2}, \eta_{b}$, and $\alpha_{1}$. As the different viscosity coefficients influence the switching of the cell with different weights, we have performed repeated fits starting from different initial sets of parameters. The results are shown in Figs. 5-10. The figures display the fitted values of the four coefficients at five different temperatures. $\alpha_{2}$ and $\alpha_{3}$ are calculated from $\gamma_{1}$ and $\gamma_{2}$ with Eq. (3). Crosses denote the

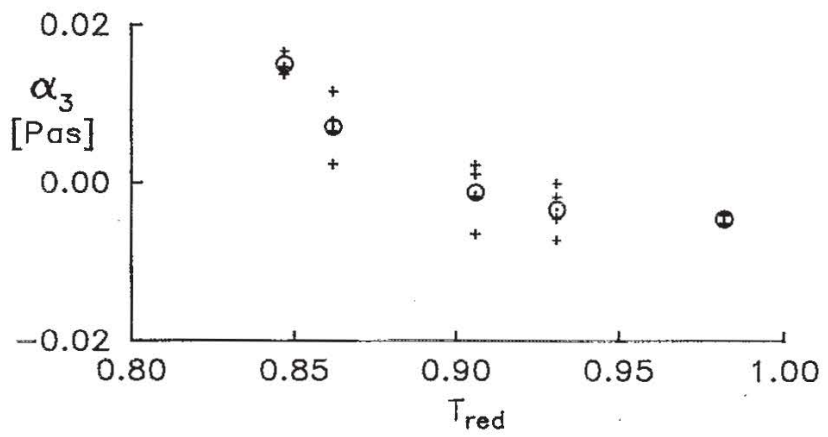

FIG. 9. Leslie viscosity coefficient $\alpha_{3}$ calculated using Eq. (3). The crosses are values obtained by fitting the calculated transmission to the measured ones for several offset voltages. The circles give the average values. 


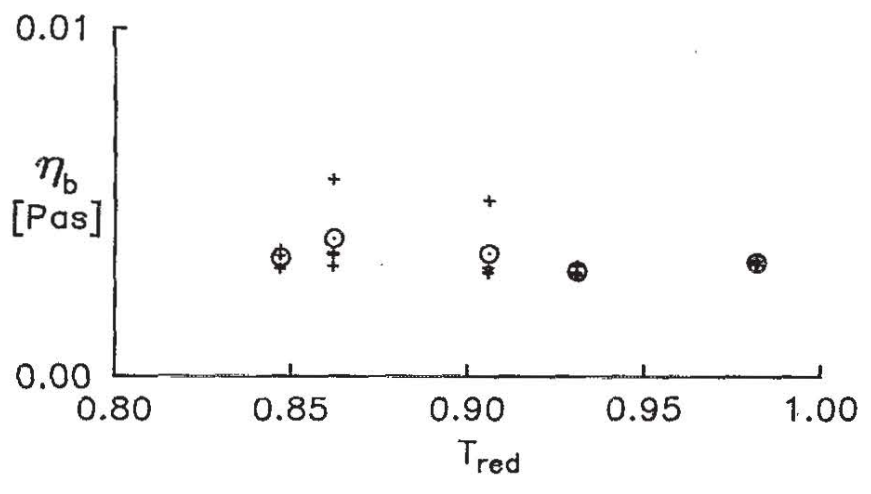

FIG. 10. Shear viscosity $\eta_{b}$. The crosses are values obtained by fitting the calculated transmission to the measured ones for several offset voltages. The circles give the average values.

results of individual fits at different offset voltages of the experimental transmission curve, circles give the average value of all fittings at one temperature. Naturally, the value of $\gamma_{1}$ is determined with high accuracy, whereas the influence of the other viscosities on the switching behavior is weaker and thus the fitted values are less stable. As expected, the determined values $\gamma_{2}=\alpha_{2}+\alpha_{3}$ are nearly equal to $-\gamma_{1}=\alpha_{3}-\alpha_{2}$ within the experimental error. In nematic liquid crystals, consisting of prolate molecules, $\alpha_{3}$ is always found to be much smaller than the other viscosities, usually by one order of magnitude (see, e.g., Ref. 3). The coefficients $\alpha_{1}$ and $\eta_{b}$ are determined with lower accuracy, the error is approximately $20 \%$.

The accuracy of the determined viscosity coefficients (except for $\gamma_{1}$ ) is certainly not very high, and there are methods that provide a more accurate determination. But if one considers the fact that there are only very few experimental data available in general, that only few experimentalists can access the specialized techniques for a direct measurement of shear viscosities in nematics, and that other techniques like light scattering give a rather indirect method for the determination of these coefficients, our method may be an acceptable alternative. It allows the direct determination of the viscosities, is based on a very simple experimental setup, and needs practically no further assumptions or simplifications. The involved elastic constants are determined with the same equipment at the same sample in an independent experiment. All viscosities are determined in one experiment, and the large amount of experimental data (amplitudes and phases of the optical transmission as a function of frequency $f_{m}$ and offset voltage $U_{0}$ ) is sufficient for a relatively stable fitting of the four independent parameters.

The sensitivity of the method might be further increased if cells with higher optical phase retardation are used (larger $n_{e}-n_{0}$ or larger $d$ ). Then, the number of slopes in the transmission curve is increased and one may have access to higher $U_{0} / U_{c}$ offset voltages where the effect of the flow coupling $\left(\alpha_{2}, \eta_{b}\right)$ is larger.

\section{ACKNOWLEDGMENT}

This work was supported by the Deutsche Forschungsgemeinschaft under grants $\operatorname{Tr} 154 / 7-1$ and Schm 902/ 2-1. The authors would like to express their thanks to $\mathrm{E}$. Merck, Darmstadt, Germany, for providing the nematic mixture ZLI 2293.

${ }^{1}$ P. G. de Gennes, The Physics of Liquid Crystals (Clarendon, Oxford, 1974).

${ }^{2}$ B. Kiebs and K. Eidner, Wiss. Zeitschr. KMU Leipzig, Naturwiss. Reihe 30, 197 (1981).

${ }^{3}$ H. Kneppe, F. Schneider, and N. K. Sharma, J. Chem. Phys. 77, 3203 (1982).

${ }^{4}$ H. H. Graf, H. Kneppe, and F. Schneider, Mol. Phys. 77, 521 (1992).

${ }^{5}$ A. F. Martins, P. Esnault, and F. Volino, Phys. Rev. Lett. 57, 1745 (1986).

${ }^{6}$ M. Miesowicz, Bull. Int. Acad. Polonia A 228 (1936); Fizyka Dielekt. i Radiospect. XI/2, 135 (1979).

${ }^{7}$ A. Scharkowski, H. Schmiedel, R. Stannarius, and E. Weisshuhn, Z. Naturforsch. 45a, 37 (1990).

${ }^{8}$ P. Pieranski, F. Brochard, and E. Guyon, J. Phys. (Paris) Colloq. 34, 35 (1973).

${ }^{9}$ F. M. Leslie, Quart. J. Mech. Appl. Math. 19, 357 (1966).

${ }^{10}$ J. L. Ericksen, Arch. Rat. Mech. Anal. 4, 231 (1960).

${ }^{11}$ C. Z. van Doorn, J. Appl. Phys. 46, 3738 (1975). 